HIAS-E-79

\title{
Fair Cake-Cutting among Families
}

\author{
Erel Segal-Halevi ${ }^{(a)}$ and Shmuel Nitzan ${ }^{(b),(c)}$
}

(a) Department of Computer Science, Ariel University, Ariel 40700, Israel

(b) Department of Economics, Bar Ilan University, Ramat Gan 52900, Ierael

(c) Hitotsubashi Institute for Advanced Study, Hitotsubashi University

November, 2018

Hitotsubashi Institute for Advanced Study, Hitotsubashi University

2-1, Naka, Kunitachi, Tokyo 186-8601, Japan

tel:+81 425808604 http://hias.ad.hit-u.ac.jp/

HIAS discussion papers can be downloaded without charge from:

http://hdl.handle.net/10086/27202

https://ideas.repec.org/s/hit/hiasdp.html

All rights reserved. 


\title{
Fair Cake-Cutting among Families
}

\author{
Erel Segal-Halevi · Shmuel Nitzan
}

\begin{abstract}
We study the fair division of a continuous resource, such as a landestate or a time-interval, among pre-specified groups of agents, such as families. Each family is given a piece of the resource and this piece is used simultaneously by all family members, while different members may have different value functions. Three ways to assess the fairness of such a division are examined. (a) *Average fairness* means that each family's share is fair according to the "family value function", defined as the arithmetic mean of the value functions of the family members. (b) *Unanimous fairness* means that all members in all families feel that their family received a fair share according to their personal value function. (c) *Democratic fairness* means that in each family, at least half the members feel that their family's share is fair. We compare these criteria based on the number of connected components in the resulting division, and based on their compatibility with Pareto-efficiency.
\end{abstract}

This research was funded in part by the following institutions: The Doctoral Fellowships of Excellence Program at Bar-Ilan University, the Mordechai and Monique Katz Graduate Fellowship Program, and the Israel Science Foundation grant 1083/13.

We are grateful to Galya Segal-Halevi, Yonatan Aumann, Avinatan Hassidim, Noga Alon, Christian Klamler, Ulle Endriss, Neill Clift and Sophie Bade for helpful discussions.

This paper started with a discussion in the MathOverflow website at http://mathoverflow.net/questions/203060/fair-cake-cutting-between-groups . We are grateful to the members who participated in the discussion: Pietro Majer, Tony Huynh and Manfred Weis. Other members of the StackExchange network contributed useful answers and ideas: Alex Ravsky, Andrew D. Hwang, BKay, Christian Elsholtz, Daniel Fischer, David K, D.W., Hurkyl, Ittay Weiss, Kittsil, Michael Albanese, Raphael Reitzig, Real, Babou, Domotor Palvolgyi (domotorp), Ian Turton (iant) and ivancho.

Erel Segal Halevi: Department of Computer Science, Ariel University, Ariel 40700, Israel, erelsgl@gmail.com.

Shmuel Nitzan: Department of Economics, Bar Ilan University, Ramat Gan 52900, Israel and Hitotsubashi Institute for Advanced Study, Hitotsubashi University. nitzans@biu.ac.il. 
Keywords fair division, cake-cutting, public good, club good, fair-share, no-envy

\section{Introduction}

Fair division of heterogeneous resources among agents with different preferences has been an important issue since Biblical times. Today it is an active area of research in the interface of computer science (Robertson and Webb 1998; Procaccia 2015) and economics (Moulin 2004). Its applications range from politics (Brams and Taylor 1996) to multi-agent systems (Chevaleyre et al 2006).

In most fair division problems, the resource is divided among $n$ individual agents, and the fairness of a division is assessed based on their individual preferences. A common fairness criterion is the fair share (FS). It requires that each agent receives a share that is at least as good as $1 / n$ of the total endowment, according to the agent's individual preferences. ${ }^{1}$

In practice, however, goods are often owned and used by groups. As an example, consider a land-estate inherited by $k$ families, a river that has to be divided among $k$ states, or the usage-time of a conference room that has to be divided among $k$ meeting groups. The resource (whether land or time) should be divided to $k$ pieces, one piece per group. Each group's share is then used by all its members simultaneously. The land-plot allotted to a family is inhabited by the entire family. The share of the river allotted to a state becomes a national park open to all its citizens. In the time-slot allotted to a group, the conference room is used by all group members. ${ }^{2}$

The happiness of each group member depends on his/her valuation of the entire share of the group. But, in each group there are different members with different valuations. The group's share can be valued by some of its members as at least $1 / k$ of the total and by others as less than $1 / k$ of the total. How, then, should the fairness of a division be assessed?

The present paper studies this question in the classic setting of cake-cutting, introduced by Steinhaus (1948). In this setting, there is a measurable space (e.g. an interval or a polygon) called the cake. The preferences of each agent are represented by a value-measure on the cake. We study three ways to assess the fairness of a division.

First, it is possible to aggregate the valuations in each family to a single family valuation. Following the utilitarian tradition (Bentham 1789), the family-valuation can be defined as the sum or (equivalently) the arithmetic average of the valuations of all family members. We call a division averagefair if it is fair according to these family valuations. In particular, a division is average-FS if every family receives a share with an average value (averaged

\footnotetext{
1 The condition of receiving at least $1 / n$ of the total endowment was introduced by Steinhaus (1948). Economists often call it fair-share guarantee (Bogomolnaia et al 2017). Computer scientists often call it proportionality (Robertson and Webb 1998).

2 In economic terms, the allotted piece becomes a "club good" (Buchanan 1965).
} 
over all family members) of at least $1 / k$ of its average value of the entire endowment.

By this definition, the family-division problem is easy to solve. Since the average of measures is itself a measure, each family can be represented by a single agent, and the problem reduces to fair division among the $k$ representatives. Classic results imply that average-FS allocations exist (Section 3).

Average fairness makes sense only when the numeric values of the agents' valuations are meaningful and they are all measured in the same units, e.g. in dollars (see chapter 3 of Moulin (2004) for some real-life examples of such situations). However, if the valuations represent individual happiness measures that cannot be put on a common scale, then their sum is meaningless, and other fairness criteria should be used.

A second option is to require that all members of every family agree that the division is fair. We call a division unanimous fair if it is fair according to every individual valuation. In particular, a division is unanimous-FS if every agent values his/her family's share as at least $1 / k$ of the total value. The advantage of this definition is that it does not need to assume that all valuations share a common scale. Even though it is a very strong requirement, we prove that unanimous-FS allocations exist (Section 4).

A disadvantage of unanimous fairness, compared to average fairness, is that unanimously-fair divisions might be highly fractioned. As an illustration, when an interval is divided, there always exists an average-FS division that is also connected - the share of each family is a single interval (Section 3). However, we prove that there might not exist connected unanimous-FS divisions. Moreover, in some cases, the number of intervals in any unanimous-FS division might be at least $n$ - the number of individual agents (Section 4). When the number of agents is large, as in the case of dividing land among states, such divisions might be impractical.

In democratic societies, decisions are almost never accepted unanimously. In fact, when the number of citizens is large, it may be impossible to attain unanimity on even the most trivial issue. The simplest decision rule in such societies is the majority rule. Inspired by this rule, we suggest a third fairness criterion. We call a division democratic fair if at least half the citizens in each family consider it fair. In particular, a division is democratic-FS if at least half the agents in each family value their family's share as at least $1 / k$ of the total.

Democratic fairness can be justified by the following process. After a division is proposed, each group conducts a referendum in which each citizen approves the division if he/she feels that the division is fair. The division is implemented only if, in every group, at least half of its members approve it.

Democratic-fairness combines some advantages of average-fairness and unanimous-fairness. It is similar to unanimous-fairness in that it does not need to assume that all valuations share a common scale. When there are $k=2$ families, it is similar to average-fairness in that it can be satisfied with connected pieces - there always exists a democratic-FS division in which each family re- 
ceives a single connected piece. An additional advantage of democratic fairness in this case is that it can be computed efficiently (Section 5). ${ }^{3}$

Although democratic-fairness might leave up to half the citizens unhappy, this may be unavoidable in real-life situations. This is understandable in light of Winston Churchil's dictum: "democracy is the worst form of government, except all the others that have been tried". 4

While the geometric requirement of having a connected division is practically important, an even more important requirement from an economic perspective is Pareto-efficiency. We prove that all three variants of fair-share are compatible with Pareto-efficiency (Section 6).

A second fairness criterion that is very common in economics is no envy (NE). In the context of individual agents, it means that each agent receives a share that is at least as good as the share of any other agent, according to the first agent's individual valuation. ${ }^{5}$ In the context of families, three variants of $\mathrm{NE}$ can be defined analogously to the three variants of fair-share: average-NE, unanimous-NE and democratic-NE (Section 7).

From a geometric perspective, these three variants behave similarly to their FS counterparts, that is:

- Connected average-NE allocations always exist;

- Connected unanimous-NE allocations are not guaranteed to exist even for two families;

- Connected democratic-NE allocations are guaranteed to exist for two but not for three or more families.

However, from an economic perspective, NE behaves differently:

- Pareto-efficient average-NE allocations always exist;

- Pareto-efficient unanimous-NE allocations are guaranteed to exist for two but not for three or more families;

- Pareto-efficient democratic-NE allocations are guaranteed to exist for two but not for five or more families (we do not know whether they always exist with three or four families).

\footnotetext{
3 In contrast, average-fair and unanimous-fair allocations cannot be computed by any finite protocol. See Remark 1.

4 A fourth fairness criterion that could be considered is individual fairness. In particular, an allocation is individually-FS if the allocation $X=\left(X_{1}, \ldots, X_{k}\right)$ admits a refinement $Y=\left(Y_{1}, \ldots, Y_{n}\right)$, where for each family $F_{j}, \cup_{i \in F_{j}} Y_{i}=X_{j}$, such that for each agent $i$, $V_{i}\left(Y_{i}\right) \geq 1 / n$. Individually-fair allocations always exist and can be found by using any classic fair division procedure on the individual agents, disregarding their families. Individualfairness makes sense if, after the division of the land among the families, each family intends to further divide its share among its members. However, often this is not the case. When an inherited land-estate is divided between two families, the members of each family intend to live and use their entire share together, rather than dividing it among them. Therefore, the happiness of each family member depends on the entire value of his family's share, rather than on the value of a potential private share he would get in a hypothetic sub-division.

5 The condition of receiving at least as much as any other agent was introduced by Gamow and Stern (1958) and Foley (1967). Economists often call it no envy (Bogomolnaia et al 2017). Computer scientists often call it envy-freeness (Robertson and Webb 1998).
} 
The paper is organized as follows. Most of the paper focuses on the fairshare criterion. Section 2 formally presents the model. Sections 3, 4 and 5 study average, unanimous and democratic fair-share divisions respectively. We study this criterion both for families with equal entitlements and for families that have different entitlements to the resource.

Section 6 studies the three variants of fair-share in combination with Paretoefficiency. Section 7 studies family fairness based on the no-envy criterion, explaining the differences between the results for fair-share and for no-envy. Finally, Section 8 compares our work to previous and ongoing related work.

\section{Model and Notation}

\subsection{Resource and agents}

In the usual cake-cutting setting, there is a resource $C$ ("cake") that has to be divided. For simplicity it is assumed that $C$ is an interval in $\mathbb{R}$. A realistic example of such a resource is time: consider a conference room that can host a single meeting at a time. It is active between 8:00 and 20:00, and this timeinterval must be divided among all those who want to use the room. Another realistic example is the shoreline of a sea or a river: while usually not a straight line, it can be easily mapped to an interval.

There is a set of agents $N=\{1, \ldots, n\}$. Each agent $i \in N$ has a value measure $V_{i}$, defined on the Borel subsets of $C$. The $V_{i}$ are assumed to be nonatomic, so that all singular points have a value of 0 to all agents. As the term measure implies, the $V_{i}$ are additive - the value of a union of two disjoint pieces is the sum of the values of the pieces. Such value functions can be viewed as having a "constant marginal utility" property (Chambers 2005). The value measures are normalized such that $\forall i: V_{i}(\emptyset)=0, V_{i}(C)=1$.

\subsection{Families and entitlements}

In our setting, there is a set of families $F=\left\{F_{1}, \ldots, F_{k}\right\}$. We use the term "family" to emphasize that the partition of agents to groups is fixed in advance and cannot be modified during the division process.

The number of agents in $F_{j}$ is denoted $n_{j}$. Each agent $i \in N$ is a member of exactly one family $F_{j} \in F$, so $n=\sum_{j=1}^{k} n_{j}$.

For each family $F_{j}$, there is a positive weight $w_{j}$ representing the entitlement of this family. The sum of all weights is one: $1=\sum_{j=1}^{k} w_{j}$.

In the simplest setting, the families have equal entitlements, i.e, for each $j \in\{1, \ldots, k\}: w_{j}=1 / k$. Equal entitlements make sense, for example, when $k$ siblings inherit their parents' estate. While an heir will probably like to take his family's preferences into account when selecting a share, each heir is entitled to $1 / k$ of the estate regardless of the size of his/her family. 
In general, each family may have a different entitlement. The entitlement of a family may depend on its size but may also depend on other factors. For example, consider several families who jointly buy a vacation apartment. The apartment can host one family at a time, so the families have to divide the year (a time-interval) among them. The entitlement of each family naturally depends on the amount of money it contributed to the purchase, rather than on the family's size. ${ }^{6}$

\subsection{Allocations and components}

An allocation is a vector of $k$ pieces, $X=\left(X_{1}, \ldots, X_{k}\right)$, one piece per family, such that the $X_{j}$ are pairwise-disjoint and $\cup_{j} X_{j}=C$.

Each piece is a finite union of intervals. We denote by $\operatorname{Comp}\left(X_{j}\right)$ the number of connected components (intervals) in the piece $X_{j}$, and by $\operatorname{ComP}(X)$ the total number of components in the allocation $\mathrm{X}$, i.e:

$$
\operatorname{Comp}(X)=\sum_{j=1}^{k} \operatorname{Comp}\left(X_{j}\right)
$$

Ideally, we would like that each piece be connected, i.e, $\forall i$ : $\operatorname{Comp}\left(X_{i}\right)=$ 1 and $\operatorname{Comp}(X)=k$. This requirement is especially meaningful when the divided resource is a time-interval or a land-resource (e.g. a river-bank), since a contiguous piece of time or land is much easier to use than a collection of disconnected patches.

However, we will show that a fair division with connected pieces is not always possible. ${ }^{7}$ In case a division with connected pieces is not possible, it is still desirable that the number of connectivity components - $\operatorname{CoMP}(X)-$ be as small as possible. When dividing an interval, the components are subintervals and their number is one plus the number of cuts. Hence, the number of components is minimized by minimizing the number of cuts (Robertson and Webb 1995; Webb 1997; Shishido and Zeng 1999; Barbanel and Brams $2004,2014)$. In a realistic, 3-dimensional world, the additional dimensions can be used to connect the components, e.g, by bridges or tunnels. Still, it is

\footnotetext{
6 See Cseh and Fleiner (2017) for a recent account of fair division among individual agents with different entitlements.

7 This impossibility appears not only in our one-dimensional theoretic model but also in practical, two-dimensional land division situations. A striking example was the IndiaBangladesh border. According to Wikipedia page India-Bangladesh enclaves, up to 2015, "Within the main body of Bangladesh were 102 enclaves of Indian territory, which in turn contained 21 Bangladeshi counter-enclaves, one of which contained an Indian countercounter-enclave... within the Indian mainland were 71 Bangladeshi enclaves, containing 3 Indian counter-enclaves". Another example is Baarle-Hertog - a Belgian municipality made of 24 separate parcels of land, most of which are exclaves in the Netherlands. For more details and examples see the Wikipedia page List of enclaves and exclaves. We are grateful to Ian Turton for the references.
} 
desirable to minimize the number of components in the original division in order to reduce the number of required bridges/tunnels. ${ }^{8}$

\subsection{Fairness criteria}

We first define the family-valuation functions:

$$
W_{j}^{\mathrm{avg}}\left(X_{j}\right)=\frac{\sum_{i \in F_{j}} V_{i}\left(X_{j}\right)}{n_{j}} \quad \text { for } j \in\{1, \ldots, k\} .
$$

Now, an allocation $X$ is called:

$$
\begin{array}{ccc}
\text { average-FS } & \text { if } \forall j \in\{1, \ldots, k\}: & W_{j}^{\mathrm{avg}}\left(X_{j}\right) \geq w_{j} ; \\
\text { unanimous-FS } & \text { if } \forall j \in\{1, \ldots, k\}: & \forall i \in F_{j}: V_{i}\left(X_{j}\right) \geq w_{j} ; \\
\text { democratic-FS } & \text { if } \forall j \in\{1, \ldots, k\}, & \\
\text { for at least half the members } i \in F_{j}: V_{i}\left(X_{j}\right) \geq w_{j} .
\end{array}
$$

A property of an allocation is called feasible if for every $k$ families and $n$ agents there exists an allocation satisfying this property. Otherwise, the property is called infeasible. In the following sections we will study the feasibility of the above fairness criteria.

Note that unanimous-FS obviously implies both average-FS and democratic-FS. The other two do not imply each other, as shown in the following example.

Example 1 Consider an interval consisting of four sub-intervals. It has to be divided between two families: (1) \{Alice,Bob,Chana\} and (2) \{David,Esther,Frank\}. The families have equal entitlements, i.e, $w_{1}=w_{2}=1 / 2$. Each member's valuation of each sub-interval is shown in the table below:

\begin{tabular}{|c|c|c|c|c|}
\hline Alice & 60 & 30 & 3 & 3 \\
\hline Bob & 50 & 40 & 3 & 3 \\
\hline Chana & 10 & 80 & 3 & 3 \\
\hline \hline David & 3 & 3 & 60 & 30 \\
\hline Esther & 3 & 3 & 60 & 30 \\
\hline Frank & 3 & 3 & 0 & 90 \\
\hline
\end{tabular}

Note that the value of the entire interval is 96 according to all agents. Therefore, FS implies that each family should get a value of at least 48 .

If the two leftmost subintervals are given to family 1 and the two rightmost subintervals are given to family 2 , then the division is unanimous-FS, since each member of each family feels that his family's share is worth 90. This division is also, of course, average-FS and democratic-FS.

\footnotetext{
8 The goal of minimizing the number of components is pursued not only in cake-cutting papers but also in real-life politics. Going back to India and Bangladesh, after many years of negotiations they finally started to exchange most of their enclaves during the years 2015-2016. This reduced the number of components from 200 to a more reasonable number.
} 
If only the single leftmost subinterval is given to family 1 and the other three are given to family 2 , then the division is still democratic-FS, since Alice and Bob feel that their family received more than 48. However, Chana feels that her family received only 10, so the division is not unanimous-FS. Moreover, the division is not average-FS since the average valuation of family 1 is only $(60+50+10) / 3=40$.

If the three leftmost subintervals are given to family 1 and only the rightmost one is given to family 2 , then the division is average-FS, since family 2 's average valuation of its share is $(30+30+90) / 3=50$. However, it is not unanimous-FS nor even democratic-FS, since David and Esther feel that their share is worth only 30 .

\section{Average fairness}

With average fairness, the family cake-cutting problem can be reduced to the classic problem of cake-cutting among individuals. This gives the following results.

Theorem 1 (a) When families have equal entitlements, average-FS with connected pieces (and $k$ components) is feasible.

(b) When families have different entitlements, average-FS with connected pieces is infeasible. Moreover, at least $2 k-1$ components may be required for an average-FS allocation.

(c) When families have different entitlements, average-FS with at most $2 k\left\lceil\log _{2} \frac{k}{2}\right\rceil+3$ components is feasible.

Proof The positive results — parts (a) and (c) — are based on the following reduction. For each family $F_{j}$, define a representative agent $A_{j}$ whose valuation is the function $W_{j}^{\text {avg }}$ defined in Subsection 2.4 above. Note that, since the $V_{i}$ are all nonatomic measures, the $k$ family-valuations $W_{j}^{\text {avg }}$ are nonatomic measures too. By classic results (Steinhaus 1948; Even and Paz 1984), when there are $k$ agents with equal entitlements, there always exists a connected FS division. As shown in a recent technical report (Segal-Halevi 2018), when there are $k$ agents with different entitlements, there always exists a FS allocation with at most $2 k \log _{2} \widehat{k}-2 \widehat{k}+2$ cuts, where $\widehat{k}:=2^{\left\lceil\log _{2} k\right\rceil}=k$ rounded up to the nearest power of two. These cuts create $2 k \log _{2} \widehat{k}-2 \widehat{k}+3$ components. By definition, such a division is an average-FS division among the families.

The negative result (b) follows immediately from an identical negative result for individual agents (Segal-Halevi 2018), by considering $k$ one-member families.

Remark 1 Fairness for individuals and average-fairness for families are equivalent only from an existential perspective; from a computational perspective they are quite different. FS division among $k$ individual agents with equal entitlements can be found by asking the agents $O(k \log k)$ queries (Even and 
Paz 1984). However, average-FS division cannot be found using a finite number of queries even when there are $k=2$ families. To see this, suppose there are two identical families, each of which has two different members with valuations $v_{A}$ and $v_{B}$. Then, a division $\left(X_{1}, X_{2}\right)$ is average-FS if and only if $\left[v_{A}\left(X_{1}\right)+v_{B}\left(X_{1}\right)\right] / 2=1 / 2$. Therefore, finding an average-FS allocation is equivalent to finding a subset $X_{1} \subseteq C$ for which the sum $v_{A}\left(X_{1}\right)+v_{B}\left(X_{1}\right)$ equals 1 . However, queries can only be sent to individual agents, and it might be impossible to find such a subset $X_{1}$ using a finite number of queries to the agents. We omit the details here since our focus is on existence. See the accompanying technical report (Segal-Halevi and Nitzan 2016) for more details.

\section{Unanimous fairness}

Before presenting our results, we note that unanimous-FS, like average-FS, can also be defined using family-valuation functions. Define:

$$
W_{j}^{\min }\left(X_{j}\right):=\min _{i \in F_{j}} V_{i}\left(X_{j}\right) \quad \text { for } j \in\{1, \ldots, k\} .
$$

Then, a division is unanimous-FS if and only if:

$$
\forall j: W_{j}^{\min }\left(X_{j}\right) \geq w_{j}
$$

However, in contrast to the functions $W^{\text {avg }}$ defined in Section 3, the functions $W^{\text {min }}$ are in general not additive. For example, suppose $C$ is an interval with three subintervals and a family has the following valuations:

\begin{tabular}{|c|c|c|c|c|}
\hline & $C_{1}$ & $C_{2}$ & $C_{3}$ & $C_{1} \cup C_{2} \cup C_{3}$ \\
\hline \hline Alice & 1 & 1 & 1 & $3=1+1+1$ \\
\hline Bob & 0 & 2 & 1 & $3=0+2+1$ \\
\hline Chana & 0 & 1 & 2 & $3=0+1+2$ \\
\hline \hline$W^{\text {min }}$ & 0 & 1 & 1 & $3>0+1+1$ \\
\hline
\end{tabular}

While the individual valuations are additive, $W^{\text {min }}$ is not additive (it is not even subadditive). Therefore, the classic results we used in Theorem 1 are inapplicable here, and different techniques are needed.

\subsection{Exact division}

Initially, we assume that the entitlements are equal, i.e: $w_{j}=1 / k$ for all $j$. We relate unanimous-FS to the problem of finding an exact division ${ }^{9}$.

Definition $1 \operatorname{Exact}(N, K)$ is the following problem. Given $N$ agents and an integer $K$, divide $C$ to $K$ pieces, such that each of the $N$ agents assigns exactly the same value to all pieces:

$$
\forall j=1, \ldots, K: \forall i=1, \ldots, N: V_{i}\left(X_{j}\right)=1 / K .
$$

\footnotetext{
9 The definition uses capital $N$ and $K$ to distinguish the parameters of exact division from the parameters of unanimous-fair division.
} 
From an economic perspective, there is little intrinsic value in the concept of exact division. However, in this section we will prove that it is closely linked to the concept of unanimously-fair division. In fact, we will prove that the existence a solution to each of these problems implies a solution to the other problem.

Denote by UnanimousFS $(n, k)$ the problem of finding a unanimous-FS division when there are $n$ agents grouped in $k$ families with equal entitlements.

\subsection{UnanimousFS $\Longrightarrow$ Exact}

Lemma 1 For every pair of integers $N \geq 1, K \geq 1$, a solution to UnanimousFS $(N(K-1)+1, K)$ implies a solution to Exact $(N, K)$.

Proof Given an instance of $\operatorname{Exact}(N, K)$ ( $N$ agents and a number $K$ of required pieces), create $K$ families. Each of the first $K-1$ families contains $N$ agents with the same valuations as the given agents. The $K$-th family contains a single agent whose valuation is the average of the $N$ given valuations:

$$
V^{*}=\frac{1}{N} \sum_{i=1}^{N} V_{i}
$$

The total number of agents in all $K$ families is $N(K-1)+1$. Use UnanimousFS $(N(K-1)+1, K)$ to find a unanimous-FS division, $X$. By definition of unanimous fairness, for each agent $i$ in family $j: V_{i}\left(X_{j}\right) \geq 1 / K$.

By construction, each of the first $K-1$ families has an agent with valuation $V_{i}$. Hence, all $N$ agents value each of the first $K-1$ pieces as at least $1 / K$ and:

$$
\forall i=1, \ldots, N: \quad \sum_{j=1}^{K-1} V_{i}\left(X_{j}\right) \geq \frac{K-1}{K} .
$$

Hence, by additivity, every agent values the $K$-th piece as at most $1 / K$ :

$$
\forall i=1, \ldots, N: \quad V_{i}\left(X_{K}\right) \leq 1 / K .
$$

The piece $X_{K}$ is given to the agent with value measure $V^{*}$, so by fair-share: $V^{*}\left(X_{K}\right) \geq 1 / K$. By construction, $V^{*}\left(X_{K}\right)$ is the average of the $V_{i}\left(X_{K}\right)$. Hence:

$$
\forall i=1, \ldots, N: \quad V_{i}\left(X_{K}\right)=1 / K .
$$

Again by additivity:

$$
\forall i=1, \ldots, N: \quad \sum_{j=1}^{K-1} V_{i}\left(X_{j}\right)=\frac{K-1}{K} .
$$


Hence, necessarily:

$$
\forall i=1, \ldots, N, \quad \forall j=1, \ldots, K-1: \quad V_{i}\left(X_{j}\right)=1 / K .
$$

So we have found an exact division and solved $\operatorname{Exact}(N, K)$ as required.

Alon (1987) proved that for every $N$ and $K$, an $\operatorname{Exact}(N, K)$ division might require at least $N(K-1)+1$ components. Combining this result with the above lemma implies the following negative result:

Theorem 2 For every $N, K$, let $n=N(K-1)+1$. A unanimous-FS division for $n$ agents grouped into $K$ families might require at least $n$ components.

In particular, unanimous-FS with connected pieces is infeasible.

\subsection{Exact $\Longrightarrow$ UnanimousFS}

Lemma 2 For every pair of integers $n \geq 2, k \geq 1$, a solution to Exact ( $n-$ $1, k)$ implies a solution to UnanimousFS $(n, k)$ for any grouping of the $n$ agents to $k$ families.

Proof Suppose we are given an instance of $\operatorname{UnanimousFS}(n, k)$, i.e, $n$ agents in $k$ families. Select $n-1$ agents arbitrarily. Use $\operatorname{Exact}(n-1, k)$ to find a partition of $C$ to $k$ pieces, such that each of the $n-1$ agents values each of these pieces as exactly $1 / k$. Ask the $n$-th agent to choose a favorite piece; by the pigeonhole principle, this value is worth at least $1 / k$ for that agent. Give that piece to the family of the $n$-th agent. Give the other $k-1$ pieces arbitrarily to the remaining $k-1$ families. The resulting division is unanimous-FS.

Alon (1987) proved that for every $N$ and $K, \operatorname{Exact}(N, K)$ has a solution with at most $N(K-1)+1$ components (at most $N(K-1)$ cuts). Combining this result with the above lemma implies the following positive result:

Theorem 3 Given $n$ agents in $k$ families with equal entitlements, a unanimous-FS division with $(n-1) \cdot(k-1)+1$ components is feasible.

For $k=2$ families, the number of components in Theorem 3 is $n$, which matches the lower bound of Theorem 2. For $k>2$ families, the number of components can be made smaller, as explained below.

\subsection{Less components: equal entitlements}

The purpose of this subsection is to find a unanimous-FS allocation with less components than the guarantee of Theorem 3, when all families have equal entitlements.

We start with an example. Assume there are $k=4$ families. By Theorem 3 , using $3(n-1)$ cuts, $C$ can be divided to 4 subsets which are considered equal by all $n$ members. But for a unanimous-FS division, it is not required 
that all members think that all pieces are equal, it is only required that all members believe that their family's share is worth at least $1 / 4$. This can be achieved as follows:

- Divide $C$ to two subsets which all $n$ agents value as exactly $1 / 2$. This is equivalent to solving $\operatorname{Exact}(n, 2)$, which by Alon (1987), can be done with at most $n$ cuts. Call the two resulting subsets West and East.

- Assign arbitrary two families to West and the other two families to East. Mark by $n_{W}$ the total number of members in the families assigned to West and by $n_{E}$ the total number of members assigned to East.

- Divide the West to two pieces which all $n_{W}$ agents value as exactly $1 / 4$; this can be done with $n_{W}$ cuts. Give a piece to each family. Divide the East similarly using $n_{E}$ cuts.

The first step requires $n$ cuts and the second step requires $n_{W}+n_{E}=n$ cuts too. Hence the total number of cuts required is only $2 n$, rather than $3 n-1$.

In fact, two cuts can be saved in each step by excluding two members (from two different families) from the exact division. These members will not think that the division is equal, but they will be allowed to choose the favorite piece for their family. Thus only $2(n-2)$ cuts are required. A simple inductive argument shows that whenever $k$ is a power of $2,\left(\log _{2} k\right) \cdot(n-k / 2)$ cuts are required.

When $k$ is not a power of 2, a result by Stromquist and Woodall (1985) can be used. They prove that, for every fraction $r \in[0,1]$, it is possible to cut a piece of $C$ such that all $n$ agents agree that its value is exactly $r$ using at most $2 n-2$ cuts. ${ }^{10}$ This can be used as follows:

- Select integers $l_{1}, l_{2} \in\{1, \ldots, k-1\}$ such that $l_{1}+l_{2}=k$.

- Apply Stromquist and Woodall (1985) with $r=l_{1} / k$ : using $2 n-4$ cuts, cut a piece $X_{1}$ that $n-1$ agents value as exactly $l_{1} / k$. This means that these $n-1$ agents value the other piece, $X_{2}$, as exactly $l_{2} / k$.

- Let the $n$-th agent choose a piece for his family; assign the other families arbitrarily such that $l_{1}$ families are assigned to piece $X_{1}$ and the other $l_{2}$ families to piece $X_{2}$.

- Recursively divide piece $X_{1}$ to its $l_{1}$ families and piece $X_{2}$ to its $l_{2}$ families.

After a finite number of recursion steps, the number of families assigned to each piece becomes 1 and the procedure ends. The number of cuts in each level of the recursion is at most $(2 n-4)$. The depth of recursion can be bounded by $\left\lceil\log _{2} k\right\rceil$ by dividing $k$ to halves (if it is even) or to almost-halves (if it is odd; i.e. take $l_{1}=(k-1) / 2$ and $\left.l_{2}=(k+1) / 2\right)$. Hence:

Theorem 4 Given $n$ agents in $k$ families with equal entitlements, a unanimous-FS division with $\left\lceil\log _{2} k\right\rceil \cdot(2 n-4)+1$ components is feasible.

\footnotetext{
10 They prove that, if $C$ is a circle, the number of connected components is $n-1$. Hence, the number of cuts is $2 n-2$. This is also true when $C$ is an interval, although the number of connected components in this case is $n$.
} 
Note that Theorem 3 and Theorem 4 both give upper bounds on the number of components required for unanimous-FS. The bound of Theorem 3 is stronger when $k$ is small and the bound of Theorem 4 is stronger when $k$ is large.

\subsection{Less components: different entitlements}

The purpose of this subsection is to find a unanimous-FS allocation with less components than the guarantee of Theorem 3, when families may have different entitlements.

When families have different entitlements, the procedure of the previous subsection cannot be used. We cannot let the $n$-th agent select a piece for his family, since the pieces are different. For example, suppose there are two families with entitlements $w_{1}=1 / 3, w_{2}=2 / 3$. We can divide $C$ to two pieces $X_{1}, X_{2}$ such that $n-1$ agents value $X_{1}$ as $1 / 3$ and $X_{2}$ as $2 / 3$. So all of them agree that $X_{1}$ should be given to family 1 and $X_{2}$ should be given to family 2 . But, the $n$-th agent might select the wrong piece for his family. Therefore, the procedure should be modified as follows.

- Select an integer $l \in\{1, \ldots, k\}$.

- Divide the families to two subsets: $F_{1}, \ldots, F_{l}$ and $F_{l+1}, \ldots, F_{k}$.

- Apply Stromquist and Woodall (1985) with $r=\sum_{j=1}^{l} w_{j}$ : using $2 n-2$ cuts, cut a piece $X_{1}$ which all $n$ agents value as exactly $\sum_{j=1}^{l} w_{j}$. This means that all $n$ agents value the other piece, $X_{2}$, as exactly $\sum_{j=l+1}^{k} w_{j}$.

- Recursively divide piece $X_{1}$ to $F_{1}, \ldots, F_{l}$ and piece $X_{2}$ to $F_{l+1}, \ldots, F_{k}$.

Here, the number of cuts in each level of the recursion is at most $(2 n-2)$. The depth of recursion can be bounded by $\left\lceil\log _{2} k\right\rceil$ by choosing $l=k / 2$ (if $k$ is even) or $l=(k-1) / 2$ (if $k$ is odd). Hence:

Theorem 5 Given $n$ agents in $k$ families with different entitlements, a unanimous-FS division with $\left\lceil\log _{2} k\right\rceil \cdot(2 n-2)+1$ components is feasible.

In concluding the analysis of unanimous-FS, recall that, even for $k=2$ families, unanimous-FS is as difficult as exact division and might require the same number of components - $n$. In the worst case, we might need to give a disjoint component to each member, which negates the concept of division to families. Therefore we now turn to the analysis of an alternative fairness criterion that yields more useful results.

\section{Democratic fairness}

Like unanimous-FS (Section 4), democratic-FS can also be defined using familyvaluation functions. Define:

$$
W_{j}^{\text {med }}\left(X_{j}\right):=\frac{\operatorname{median}_{i \in F_{j}} V_{i}\left(X_{j}\right)}{n_{j}} \quad \text { for } j \in\{1, \ldots, k\} .
$$


A division is democratic-FS if and only if:

$$
\forall j: W_{j}^{\text {med }}\left(X_{j}\right) \geq w_{j}
$$

However, the $W^{\text {med }}$ functions are not additive, ${ }^{11}$ so again the classic results referred to in Theorem 1 are inapplicable.

\subsection{Two families: a division procedure}

We start with a positive result for two families with equal entitlements, which shows that democratic-FS is substantially easier than unanimous-FS.

Theorem 6 When there are $k=2$ families with equal entitlements, democratic-FS with connected pieces is feasible. Moreover, it can be found by an efficient algorithm.

Proof The proof is given by Algorithm 1. It finds a democratic-FS division between two families. For each family, a location $M_{j}$ is calculated such that, if $C$ is cut at $M_{j}$, half the members value the interval $\left[0, M_{j}\right]$ as at least $1 / 2$ and the other half value the interval $\left[M_{j}, 1\right]$ as at least $1 / 2$. Then, $C$ is cut between the two family medians, and each family receives the piece containing its own median. By construction, at least half the members in each family value their family's share as at least $1 / 2$, so the division is democratic-FS. Each family receives a single connected piece.

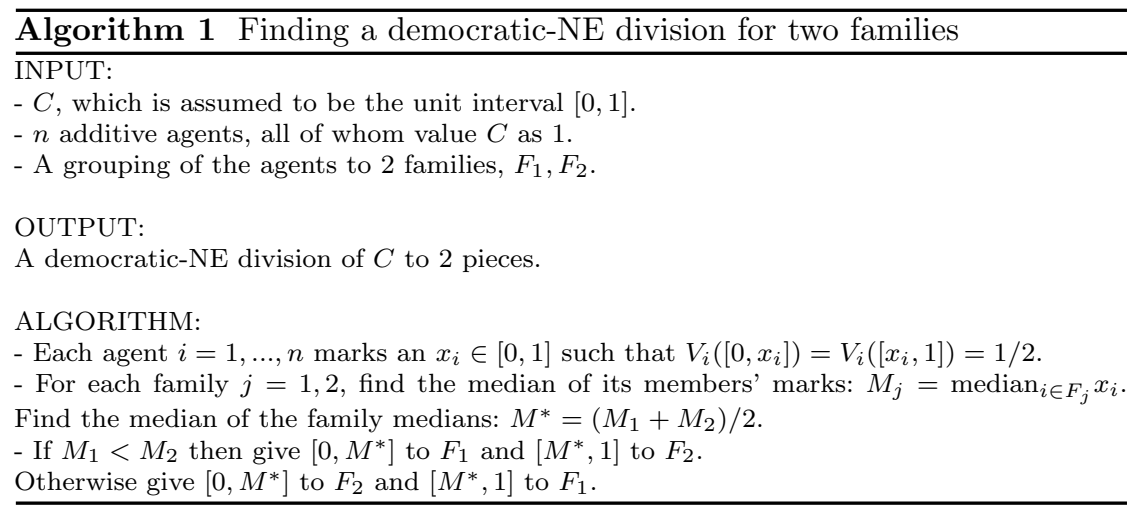

Unfortunately, this positive result is not applicable when there are more than two families, as shown in the following subsection.

11 See the example in the beginning of Section 4 . In that example $W^{\text {med }}$ is identical to $W^{\mathrm{min}}$. 


\subsection{Three or more families: an impossibility result}

This subsection presents a lower bound on the number of components required for a democratic-fair division. The lower bound holds not only for FS but even for a much weaker fairness notion called positivity.

Given a specific division of $C$ among families, define a zero agent as an agent who values his family's share as 0 and a positive agent as an agent who believes his family received a share with a positive value. Note that FS implies positivity but not vice-versa. The following lower bound holds even for positivity, hence it also holds for FS.

Lemma 3 Assume there are $n=m k$ agents, divided into $k$ families with $m$ members in each family. To guarantee that at least $q$ members in each family are positive, the total number of components may need to be at least:

$$
k \cdot \frac{k q-m}{k-1}
$$

Proof Number the families by $j=0, \ldots, k-1$ and the members in each family by $i=0, \ldots, m-1$. Assume that $C$ is the interval $[0, m k]$. In each family $j$, each member $i$ wants only the following interval: $(i k+j, i k+j+1)$. Thus there is no overlap between desired intervals of different members. The table below illustrates the construction for $k=2, m=3$. The families are \{Alice,Bob,Chana\} and $\{$ David,Esther,Frank $\}$ :

\begin{tabular}{|c|c|c|c|c|c|c|}
\hline Alice & 1 & 0 & 0 & 0 & 0 & 0 \\
\hline Bob & 0 & 0 & 1 & 0 & 0 & 0 \\
\hline Chana & 0 & 0 & 0 & 0 & 1 & 0 \\
\hline \hline David & 0 & 1 & 0 & 0 & 0 & 0 \\
\hline Esther & 0 & 0 & 0 & 1 & 0 & 0 \\
\hline Frank & 0 & 0 & 0 & 0 & 0 & 1 \\
\hline
\end{tabular}

Suppose the piece $X_{j}$ (the piece given to family $j$ ) is made of $l \geq 1$ components. We can make $l$ members of $F_{j}$ positive using $l$ intervals of positive length inside their desired areas. However, if $q>l$, we also have to make the remaining $q-l$ members positive. For this, we have to extend $q-l$ intervals to length $k$. Each such extension totally covers the desired area of one member in each of the other families. Overall, each family creates $q-l$ zero members in each of the other families. The number of zero members in each family is thus $(k-1)(q-l)$. Adding the $q$ members which must be positive in each family, we get the following necessary condition: $(k-1)(q-l)+q \leq m$. This is equivalent to:

$$
l \geq \frac{k q-m}{k-1}
$$

The total number of components is $k \cdot l$, which is at least equal to the expression stated in the Lemma. 
In other words, if we want at least a fraction $h$ of the members in each family to have a non-zero utility, the number of components might have to be at least:

$$
k \cdot \frac{k h m-m}{k-1}=n \cdot \frac{h k-1}{k-1}
$$

In a unanimous-FS division $h=1$, so the number of components is at least $n$, which coincides with the lower bound of Theorem 2 . In a democratic-FS $h \geq 1 / 2$ so we get the following negative result:

Theorem 7 In a democratic-FS division with $n$ agents grouped into $k$ families, the number of components may need to be at least

$$
n \cdot \frac{k / 2-1}{k-1}
$$

Note that for $k=2$ the lower bound is 0 , and indeed we already saw that in this case a connected allocation is feasible.

Lemma 3 has another interesting corollary. Suppose we have $k \geq 3$ but still insist that the division be connected. We already know that we cannot guarantee that $1 / 2$ the agents in each group be positive. But there is an even stronger impossibility result.

Theorem 8 When dividing a cake among $k$ families, for every constant fraction $h>1 / k$, it may be impossible to find a connected division where at least a fraction $h$ of the agents in each group are positive.

Proof By Lemma 3 the number of components should be at least $n \cdot \frac{h k-1}{k-1}$. Since $h>1 / k$, for sufficiently large $n$ the number of components is larger than $k$, so the division cannot be connected.

The fraction $1 / k$ in Theorem 8 is tight:

Theorem 9 For every integer $k \geq 2$, there exists a connected division among $k$ families, that is FS for at least $1 / k$ of the members in each family.

Proof The Dubins-Spanier moving-knife protocol (Dubins and Spanier 1961) can be adapted to families as follows. A knife moves continuously over the cake from left to right. Whenever in a certain family at least $1 / k$ of its members believe that the cake to the left of the knife is worth at least $1 / k$, they shout "stop", the cake is cut at the knife location, and the shouting family receives the cake to its left (the division is now FS for $1 / k$ the members in this family). In the other $k-1$ families, at least $(k-1) / k$ of the members believe the remaining cake is worth at least $(k-1) / k$ of its original value; by dividing the remaining cake recursively using the same procedure, they division is FS for $1 / k$ of their members too. 
5.3 Three or more families: positive results

Suppose we do want a democratic-FS division for three or more families. How many components are sufficient?

As a first positive result, we can use Theorem 5 , substituting $n / 2$ instead of $n$ : select half of the members in each family arbitrarily, then find a division which is unanimous-FS for them while ignoring all other members. This leads to:

Theorem 10 Given $n$ agents in $k$ families with different entitlements, democratic-FS with $\left\lceil\log _{2} k\right\rceil \cdot(n-2)+1$ components is feasible.

However, for families with equal entitlements we can do much better. Algorithm 2 generalizes Algorithm 1 for any number of families:

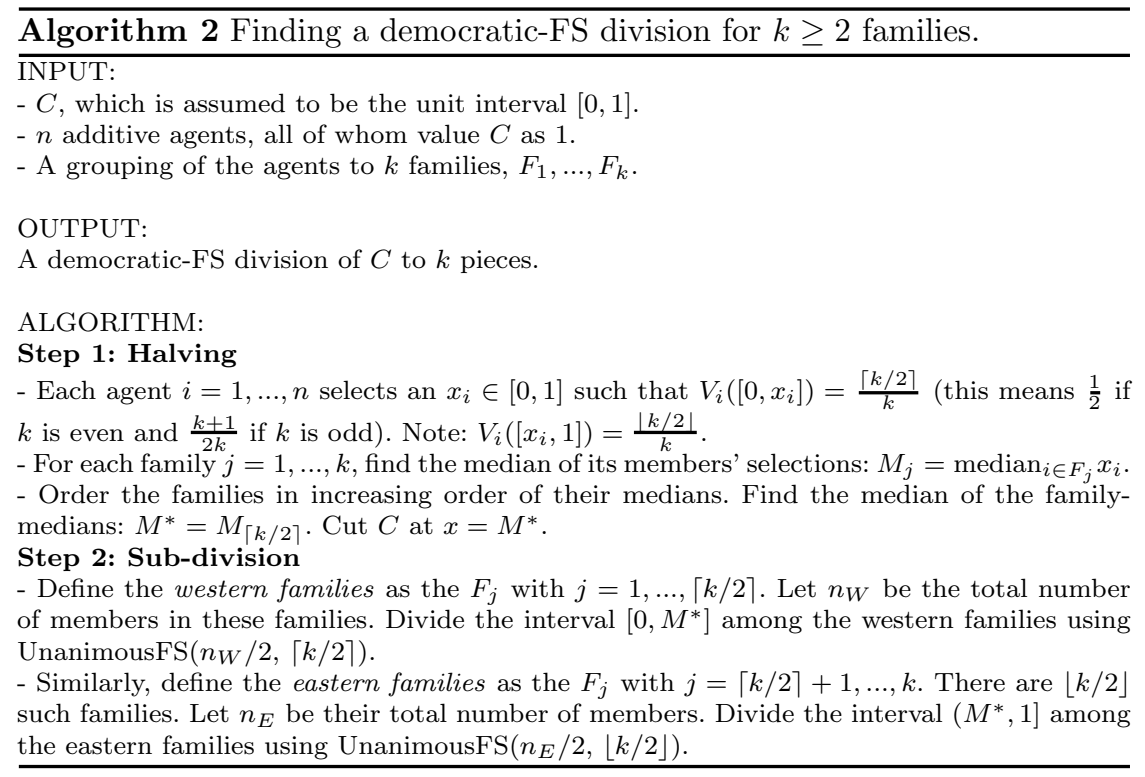

The algorithm works in two steps.

Step 1: Halving. For each family, a location $M_{j}$ is calculated such that, if $C$ is cut at $M_{j}$, half the family members value the interval $\left[0, M_{j}\right]$ as at least $\frac{[k / 2]}{k}$ and the other half value the interval $\left[M_{j}, 1\right]$ as at least $\frac{\lfloor k / 2\rfloor}{k}$. Then, $C$ is cut in $M^{*}$ - the median of the family medians. The $\lceil k / 2\rceil$ "western families" — for which $M_{j} \leq M^{*}$ - are assigned to the western interval of $C-\left[0, M^{*}\right]$. By construction, at least half the members in each of the western families value $\left[0, M^{*}\right]$ as at least $\frac{\lceil k / 2\rceil}{k}$. We say that these members are "happy". Similarly, the $\lfloor k / 2\rfloor$ eastern families - for which $M_{j} \geq M^{*}$ - are assigned to the eastern interval $\left(M^{*}, 1\right]$; at least half the members in each of these families are "happy", i.e, value the interval $\left(M^{*}, 1\right]$ as at least $\frac{|k / 2|}{k}$. 
If there are only two families $(k=2)$, then we are done: there is exactly one western family and one eastern family $(\lceil k / 2\rceil=\lfloor k / 2\rfloor=1)$. For each family $j \in\{1,2\}$, at least half the members of each family value their family's share as at least $1 / 2$. Hence, the allocation of $X_{j}$ to family $j$ is democraticFS.

If there are more than two families $(k>2)$, an additional step is required.

Step 2: Sub-division. Each of the two sub-intervals should be further divided to the families assigned to it. In each family $F_{j}$, at least $n_{j} / 2$ members are happy. So for each $F_{j}$, select exactly $n_{j} / 2$ members who are happy. Our goal now is to make sure that these agents remain happy. This can be done using a unanimous-FS allocation, where only $n_{j} / 2$ happy members in each family (hence $n / 2$ members overall) are counted.

The unanimous-FS allocation guarantees that every western-happy-member believes that his family's share is worth at least $\frac{\lceil k / 2\rceil}{k} \cdot \frac{1}{\lceil k / 2\rceil}=\frac{1}{k}$. Similarly, every eastern-happy-member believes that his family's share is worth at least $\frac{\lfloor k / 2\rfloor}{k} \cdot \frac{1}{\lfloor k / 2\rfloor}=\frac{1}{k}$. Hence, the resulting division is democratic-FS.

We now calculate the number of components in the resulting division. One cut is required for the halving step. For the unanimous-FS division of the western interval, the number of required cuts is at most $(\lceil k / 2\rceil-1) \cdot\left(n_{W} / 2-1\right)$ by Theorem 3 , and at most $\left\lceil\log _{2}\lceil k / 2\rceil\right\rceil \cdot\left(n_{W}-4\right)$ by Theorem 4 . Similarly, for the eastern interval the number of required cuts is at most the minimum of $(\lfloor k / 2\rfloor-1) \cdot\left(n_{E} / 2-1\right)$ and $\left\lceil\log _{2}\lfloor k / 2\rfloor\right\rceil \cdot\left(n_{E}-4\right)$. The total number of cuts is thus at most $1+(\lceil k / 2\rceil-1) \cdot(n / 2-2)$ and at most $1+\left\lceil\log _{2}\lceil k / 2\rceil\right\rceil \cdot(n-8)$. The total number of components is larger by one. To conclude:

Theorem 11 Given $n$ agents in $k$ families with equal entitlements, democratic-FS is feasible, and the number of required components is at most:

$$
\min \left(2+(\lceil k / 2\rceil-1) \cdot(n / 2-2) \quad, \quad 2+\left\lceil\log _{2}\lceil k / 2\rceil\right\rceil \cdot(n-8)\right) .
$$

\subsection{Comparison and Open Questions}

Table 1 compares the three variants of FS, focusing on families with equal entitlements. Recall that $n$ is the total number of agents in all families.

The case of $k=2$ families is well-understood. The results for all fairness criteria are tight: by all fairness definitions, we know that a fair division exists with the smallest possible number of connectivity components.

The case of $k>2$ families opens some questions:

- Is unanimous-FS with $n$ components feasible for all $k$ ? (particularly, with $k=3$ families, is the number of required components $n$ as in the lower bound, or $2 n-1$ as in the upper bound?).

- Is democratic-FS with $n \cdot \frac{k / 2-1}{k-1}$ components feasible for all $k$ ? (particularly, with $k=3$ families, is the number of required components $n / 4$ as in the lower bound, or $n / 2$ as in the upper bound?). 


\begin{tabular}{|c|c|c|c|}
\hline \multirow{2}{*}{ Fairness } & \multirow{2}{*}{$\begin{array}{c}\text { \#Families } \\
(k)\end{array}$} & \multicolumn{2}{|c|}{ \#Connectivity Components } \\
\hline & & Lower & Upper \\
\hline average-FS (Sec. 3) & $k$ & $k$ & $k$ (connected) \\
\hline \multirow[t]{4}{*}{ unanimous-FS } & 2 & $\bar{n}$ & $n$ \\
\hline & 3 & $n$ & $2 n-1$ \\
\hline & 4 & $\bar{n}$ & $2 n-3$ \\
\hline & $k$ & $n$ & $\begin{array}{c}\min \left(1+\left|\log _{2} k\right| \cdot(2 n-4),\right. \\
(k-1) \cdot(n-1)+1)\end{array}$ \\
\hline \multirow[t]{4}{*}{ democratic-FS } & 2 & 2 & $2($ connected $)$ \\
\hline & 3 & $n / 4$ & $n / 2$ \\
\hline & 4 & $n / 3$ & $n / 2$ \\
\hline & $k$ & $n \cdot \frac{k / 2-1}{k-1}$ & $\begin{array}{c}\min \left(2+\left|\log _{2}\right| k / 2|| \cdot(n-8)\right. \\
2+(\lceil k / 2\rceil-1) \cdot(n / 2-2))\end{array}$ \\
\hline
\end{tabular}

Table 1: Number of components required for a fair-share division in various situations.

The case of different entitlements is much less understood even for individual agents (Segal-Halevi, 2018), let alone for families.

What fairness notion is the most practical? The table shows that it depends on the total number of agents $(n)$. When $n$ is small (as is common when dividing an estate among heirs), it is reasonable to try to attain a unanimouslyfair division. However, when $n$ is large (as is common when dividing disputed lands among states), unanimous fairness quickly becomes impractical, as the number of components might grow linearly with $n$. In this case, we must settle for a weaker fairness criterion. When $k=2$, we can find a democratically-fair allocation that is also connected. When $k>2$, democratic fairness too might be impractical, and we may have to settle for average-fairness.

\section{Pareto-efficiency}

So far, we studied the compatibility of fairness criteria with a geometric requirement - reducing the number of connectivity components. In this section we replace the geometric requirement with an economic requirement - Pareto efficiency. An allocation is called Pareto-efficient (PE) if no other allocation is weakly better for all individual agents and strictly better for some individual agents. Fortunately, PE is compatible even with the strongest variant of the fair-share criterion:

Theorem 12 There always exists an allocation that is both PE and unanimous-FS (hence also average-FS and democratic-FS).

Proof We use a famous theorem of Dubins and Spanier (1961), which is a special case of a measure-theoretic theorem by Dvoretzky et al (1951).

For every partition $X$ of $C$ to $k$ pieces, let $M(X)$ be its value-matrix an $n$-by- $k$ matrix $M$ where $\forall i \in\{1, \ldots, n\}, \forall j \in\{1, \ldots, k\}: M_{i, j}=V_{i}\left(X_{j}\right)$. Let $\mathbb{M}_{C}$ be the set of all matrices that correspond to such partitions:

$$
\mathbb{M}_{C}:=\{M(X) \mid X \text { is a partition of } C \text { to } k \text { pieces }\}
$$


Theorem 1 of Dubins and Spanier (1961) implies that the set $\mathbb{M}_{C}$ is compact. Define a second set of matrices representing the unanimous-FS condition:

$$
\mathbb{M}_{F S}:=\left\{M \text { is an } n \times k \text { matrix } \mid \forall j \in\{1, \ldots, k\}: \forall i \in F_{j}: M_{i, j} \geq 1 / k\right\}
$$

Finally, define $\mathbb{M}_{C F S}:=\mathbb{M}_{C} \cap \mathbb{M}_{F S}$. This set represents all value-matrices of allocations of $C$ that are unanimous-FS. By Theorem 3, $\mathbb{M}_{C F S}$ is non-empty. Since $\mathbb{M}_{C}$ is compact and $\mathbb{M}_{F S}$ is closed, their intersection $\mathbb{M}_{C F S}$ is compact.

Define the following function $U: \mathbb{M}_{C F S} \rightarrow \mathbb{R}$ :

$$
U(M):=\prod_{j=1}^{k} \prod_{i \in F_{j}} M_{i, j}
$$

This is a continuous function, so it has a maximum point in $\mathbb{M}_{C F S}$; let's call it $M^{*}$. This matrix corresponds to an allocation $X^{*}$ that maximizes, among all unanimous-FS allocations, the product of valuations of all agents: $\prod_{j=1}^{k} \prod_{i \in F_{j}} V_{i}\left(X_{j}\right)$. This product is strictly increasing with the value of each agent $i \in N$, so the allocation $X^{*}$ is Pareto-efficient in the set $\mathbb{M}_{C F S}$. Since every Pareto-improvement of an allocation in $\mathbb{M}_{C F S}$ is also in $\mathbb{M}_{C F S}$, the allocation $X^{*}$ is also Pareto-efficient in general.

\section{No Envy}

So far, we used fair-share (FS) as our individual fairness criterion. Another criterion that is very common in economics is no-envy (NE). We study this criterion for families with equal entitlements.

Analogously to the definitions in subsection 2.4, we call an allocation $X$ :

$$
\begin{aligned}
\text { average-NE } & \text { if } \forall j, j^{\prime} \in\{1, \ldots, k\}: \quad W_{j}^{\text {avg }}\left(X_{j}\right) \geq W_{j}^{\text {avg }}\left(X_{j^{\prime}}\right) ; \\
\text { unanimous-NE } & \text { if } \forall j, j^{\prime} \in\{1, \ldots, k\}: \quad \forall i \in F_{j}: V_{i}\left(X_{j}\right) \geq V_{i}\left(X_{j^{\prime}}\right) ; \\
\text { democratic-NE } & \text { if } \forall j, j^{\prime} \in\{1, \ldots, k\},
\end{aligned}
$$

for at least half the members $i \in F_{j}: V_{i}\left(X_{j}\right) \geq V_{i}\left(X_{j^{\prime}}\right)$.

With individual agents, it is well known that NE implies FS (with equal entitlements). With two individual agents, NE and FS are equivalent. The same implications are true with families. Each variant of NE implies the corresponding variant of FS. ${ }^{12}$ When there are $k=2$ families, each variant of NE is equivalent to the corresponding variant of FS. ${ }^{13}$

\footnotetext{
12 Suppose an agent $i \in F_{j}$ thinks the division is not FS. This means that $V_{i}\left(X_{j}\right)<1 / k$. But, the sum of all weights equals 1 which equals the sum of the values of all pieces. Therefore there must be some $j^{\prime} \neq j$ for which: $V_{i}\left(X_{j^{\prime}}\right)>1 / k$. Hence, $V_{i}\left(X_{j}\right)<V_{i}\left(X_{j^{\prime}}\right)$, so agent $i$ thinks the division is not NE.

13 Suppose an agent $i \in F_{j}$ thinks the division is FS. This means that $V_{i}\left(X_{j}\right) \geq 1 / 2$. By additivity, for the other family $j^{\prime} \neq j, V_{i}\left(X_{j^{\prime}}\right) \leq 1 / 2$. Hence $V_{i}\left(X_{j}\right) \geq V_{i}\left(X_{j^{\prime}}\right)$, so agent $i$ thinks the division is NE.
} 
Most of our results for FS with equal entitlements are also valid for NE. For average-NE, we can use classic results proving the existence of NE allocations with connected pieces among individual agents (Stromquist 1980; Su 1999). Applying the same reduction as in Theorem 1 we get:

Theorem 1' For any $k$ families, average-NE with connected pieces is feasible.

Since NE implies FS, the negative results are still valid:

Theorem 2' For every $N, K$, let $n=N(K-1)+1$. A unanimous- $N E$ division for $n$ agents grouped into $K$ families might require at least $n$ components.

Some positive results remain valid too. Lemma 2 is based on an exact division. Therefore it holds, with the same proof, even if we replace unanimousFS with unanimous-NE. Therefore:

Theorem 3' Given $n$ agents in $k$ families, a unanimous-NE division with $(n-1) \cdot(k-1)+1$ components is feasible.

However, the recursive-halving procedure of Theorem 4 cannot be used here. Suppose we divide $C$ to two subsets, West and East, which all $n$ agents value as exactly $1 / 2$. Then, we assign arbitrary $k / 2$ families to West and the other families to East. We find an exact division of the West among the western families and an exact division of the East among the eastern families. While this division satisfies FS, it does not satisfy NE, since the agents in the west might envy families in the east and vice versa. Therefore, while the number of components required for unanimous-FS division is in $O(n \log k)$, the best we can currently say about the number of components required for unanimous$\mathrm{NE}$ is that it is in $O(n k)$.

With two families FS implies NE, so the following positive result holds:

Theorem 6' When there are $k=2$ families, democratic-NE with connected pieces is feasible. Moreover, it can be found by an efficient algorithm.

Similarly, the negative results for democratic-FS in Theorems 7 and 8 are equally valid for democratic-NE. The positive result of Theorem 9 holds for NE too:

Theorem 9' For every integer $k \geq 2$, there exists a connected division among $k$ families, that is $N E$ for at least $1 / k$ of the members in each family.

Proof Su (1999) presents a procedure (attributed to Simmons) for finding a connected NE division among $k$ individual agents. It is based on presenting various connected partitions to the agents, and asking each agent which of the $k$ pieces is the best. He proves that there exists a partition in which each agent gives a different answer; that partition corresponds to a no-envy allocation. He also shows a procedure for finding a sequence of partitions that converges (after possibly infinitely many steps) to that no-envy allocation.

The Simmons-Su procedure can be adapted to families in the following way. Whenever a family is asked "which of the $k$ pieces is better?", it answers 


\begin{tabular}{|c|c||c|c|}
\hline \multirow{2}{*}{ Fairness } & \multicolumn{2}{|c||}{ \#Families $(k)$} & \multicolumn{2}{|c|}{ \#Connectivity Components } \\
\cline { 2 - 4 } & & Lower bound & Upper bound \\
\hline \hline average-NE & Any & $k$ & $k$ (connected) \\
\hline \hline \multirow{3}{*}{$\begin{array}{c}\text { unanimous-NE } \\
(\text { Sec. 4) }\end{array}$} & 2 & $n$ & $n$ \\
\cline { 2 - 4 } & 3 & $n$ & $2 n-1$ \\
\cline { 2 - 4 } & 4 & $n$ & $3 n-2$ \\
\hline \hline \multirow{3}{*}{$\begin{array}{c}\text { democratic-NE } \\
\text { (Sec. 5) }\end{array}$} & Any & $n$ & $2(k-1) \cdot(n-1)+1$ \\
\cline { 2 - 4 } & 2 & 2 & $n / 2+2$ \\
\cline { 2 - 4 } & 3 & $n / 4$ & $n+1$ \\
\cline { 2 - 4 } & Any & $n \cdot \frac{k / 2-1}{k-1}$ & $n \cdot(k-2) / 2+2$ \\
\hline
\end{tabular}

Table 2: Number of components required for a no-envy division in various situations.

by doing a plurality voting among its members. Then, in the final division, each family receives a piece that is considered the best by a plurality of its members, which is at least a fraction $1 / k$ of its members. Therefore, at least $1 / k$ of each family's members feel that the allocation has no envy.

Theorem 11 about democratic-FS does not hold as-is for democratic-NE, but it can be adapted by adapting Algorithm 2. Step 1 - the halving step remains the same. Step 2 - the subdivision step - should be modified to use an exact division, as follows:

- Using $\operatorname{Exact}(n / 2,\lceil k / 2\rceil)$, find an exact division of the interval $\left[0, M^{*}\right]$ into $\lceil k / 2\rceil$ pieces, such that all $n / 2$ happy agents find the pieces equal.

Assign these pieces to the western families - the $F_{j}$ with $j=1, \ldots,\lceil k / 2\rceil$.

- Using $\operatorname{Exact}(n / 2,\lfloor k / 2\rfloor)$, find an exact division of the interval $\left(M^{*}, 1\right]$ into $\lfloor k / 2\rfloor$ pieces, such that all $n / 2$ happy agents find the pieces equal.

Assign the pieces to the eastern families $-F_{j}$ with $j=\lceil k / 2\rceil+1, \ldots, k$.

The halving step requires a single cut. The two exact divisions require $(n / 2) \cdot(k-2)$ cuts. Therefore the total number of components is $n(k-2) / 2+2$ :

Theorem 11' Given $n$ agents in $k$ families with equal entitlements, democratic-NE with at most $n(k-2) / 2+2$ components is feasible.

Table 2 summarizes our results for no-envy division and shows some remaining gaps.

We now consider the combination of no-envy with Pareto-efficiency. Some of our positive results from Section 6 are still valid:

Theorem 12' (a) With $k=2$ families, there always exists an allocation that is both PE and unanimous-NE (hence also average-NE and democratic-NE).

(b) With any number of families, there always exists an allocation that is $P E$ and average- $N E$.

Proof (a) With $k=2$ families, NE and FS are equivalent, so this follows directly from Theorem 12 . 
(b) We use the same reduction as in Theorem 1 and the same compactness argument as in Theorem 12 . For each family $F_{j}$, define a representative agent $A_{j}$ whose valuation is $W_{j}^{\text {avg }}$. There exists an allocation $X^{*}$ that maximizes the product of valuations of the representatives: $\prod_{j=1}^{k} W_{j}^{\mathrm{avg}}\left(X_{j}\right)$.

Segal-Halevi and Sziklai (2018, Section 5) prove, in the setting of cakecutting among individuals, that every allocation maximizing the product of values has no envy. Therefore, in the allocation $X^{*}$, there is no envy among the representatives. By definition of average-NE, $X^{*}$ is an average-NE allocation among the families.

The product $\prod_{j=1}^{k} W_{j}^{\mathrm{avg}}\left(X_{j}\right)$ is strictly increasing with the value of each individual agent $i \in N$. Therefore, the allocation $X^{*}$ maximizing this product is Pareto-efficient.

In contrast to these positive results, Pareto-efficiency is incompatible with unanimous-NE and democratic-NE.

The incompatibility between PE and unanimous-NE appears even when we take a minimal step forward from the case of two families: there are three families, only one of which is a couple and the other two are singles.

Theorem 13 With three or more families, there might be no allocation that is both PE and unanimous-NE.

Proof The proof is based on an example used by Bade and Segal-Halevi (2018) in the context of fair division of homogeneous goods. $C$ is an interval composed of two sub-intervals $Y$ and $Z$ of length 1 . $C$ has to be divided among three families — a couple and two singles — with the following valuations:

\begin{tabular}{|c|c|c|}
\hline & $\mathrm{Y}$ & $\mathrm{Z}$ \\
\hline \hline Alice & 1 & 1 \\
\hline George & 7 & 1 \\
\hline \hline Bob & 2 & 1 \\
\hline \hline Esther & 5 & 1 \\
\hline
\end{tabular}

Suppose that we have a unanimous-NE allocation of $C$ among the three families. Denote by $Y_{A G}, Z_{A G}$ the lengths of $Y, Z$ given to Alice+George, and 
similarly $Y_{B}, Z_{B}, Y_{E}, Z_{E}$ are the lengths given to Bob and Esther. Then:

$\begin{array}{rr}7 Y_{A G}+Z_{A G} \geq 7 Y_{B}+Z_{B} & \text { (George does not envy Bob) } \\ 2 Y_{B}+Z_{B} \geq 2 Y_{A G}+Z_{A G} & \text { (Bob does not envy George) } \\ (7-2) Y_{A G} \geq(7-2) Y_{B} & \text { (from the above inequalities) } \\ (*) & \\ Y_{A G} \geq Y_{B} & \text { (Alice does not envy Bob) } \\ 1 Y_{A G}+Z_{A G} \geq 1 Y_{B}+Z_{B} & \text { (Bob does not envy Alice) } \\ 2 Y_{B}+Z_{B} \geq 2 Y_{A G}+Z_{A G} & \text { (from the above inequalities) } \\ (1-2) Y_{A G} \geq(1-2) Y_{B} & \\ Y_{A G} \leq Y_{B} & \\ Y_{A G}=Y_{B} & \\ \Longrightarrow Z_{A G}=Z_{B} & \text { (Bob and Alice+George do not envy) }\end{array}$

We proved that, in any unanimous-NE allocation, the share given to Alice+George is identical to the share given to Bob (i.e, the same lengths of both subintervals). The proof does not depend on the exact valuation functions - it only depends on the fact that $1<2<7$, i.e, Bob's valuation of $Y$ is strictly between Alice's and George's valuations. Hence, exactly the same proof works for Esther, i.e: $Y_{A G}=Y_{E}$ and $Z_{A G}=Z_{E}$. Therefore, the shares given to all three families are identical.

We now prove that this allocation cannot be PE. We consider three cases.

- Case 1: $Y_{B}=0$. Then also $Y_{E}=Y_{A G}=0$ so $Y$ remains unallocated and the allocation is not PE.

- Case 2: $Z_{E}=0$. Then also $Z_{B}=Z_{A G}=0$ so $Z$ remains unallocated and the allocation is not $\mathrm{PE}$.

- Case 3: $Y_{B}$ and $Z_{E}$ are positive. Let $\epsilon=\min \left(Y_{B}, Z_{E} / 3\right)$. Suppose that Bob gives $\epsilon$ of his $Y$ to Esther, and gets in exchange $3 \epsilon$ of her $Z$. Then, Bob's value increases by $3 \epsilon-2 \epsilon$; Esther's value increases by $5 \epsilon-3 \epsilon$; and the values of Alice and George are unchanged. This means that the original allocation was not Pareto-efficient.

Weakening unanimous-NE to democratic-NE does not help when there are 5 or more families.

Theorem 14 With five or more families, there might be no allocation that is both $P E$ and democratic-NE.

Proof The proof is based on an extension of the example of Theorem 13, where there are five families — one triplet and four singles — with the valuations: 


\begin{tabular}{|c|c|c|}
\hline & $\mathrm{Y}$ & $\mathrm{Z}$ \\
\hline \hline Alice & $1 / 4$ & 1 \\
\hline Dina & 1 & 1 \\
\hline George & 4 & 1 \\
\hline \hline Bob & $1 / 2$ & 1 \\
\hline \hline Chana & $1 / 3$ & 1 \\
\hline \hline \hline Esther & 2 & 1 \\
\hline \hline Frank & 3 & 1 \\
\hline \hline
\end{tabular}

Suppose that we have a democratic-NE allocation of $C$ among the families. By definition of democratic-NE, all singles must not feel any envy. Moreover, in the first family, at least two members must not feel any envy. There are three options for the identity of these non-envious members.

Option A: Alice and Dina feel no envy. We consider Bob and Chana. The value of $Y$ for each of them is strictly between the value of $Y$ for Alice and the value of $Y$ for Dina. Therefore, similar arguments as in the proof of Theorem 13 imply that the three allocations of Chana, Bob, and Alice+Dina+George are identical. Now there are three cases:

- Case A1: $Y_{C}=0$. Then also $Y_{B}=Y_{A D G}=0$. Each of Chana, Bob, and Alice+Dina+George receive at most $1 / 3$ of $Z$, so Dina's value is at most $1 / 3$. Since Dina does not envy Esther and Frank, each of them must receive at most $1 / 3$ of $Y$. This means that at least $1 / 3$ of $Y$ remains unallocated, so the allocation is not PE.

- Case A2: $Z_{B}=0$. Then also $Z_{C}=Z_{A D G}=0$. Again Dina's value is at most $1 / 3$, so Esther and Frank must receive at most $1 / 3$ of $Z$, so at least $1 / 3$ of $Z$ remains unallocated, so the allocation is not $\mathrm{PE}$.

- Case A3: $Y_{C}$ and $Z_{B}$ are positive. Then, Bob can give $\epsilon / 2.5$ of his $Z$ to Chana in exchange for $\epsilon$ of her $Y$ (for some small $\epsilon>0$ ) and attain a Pareto improvement, so the original allocation is not $\mathrm{PE}$.

Option B: George and Dina feel no envy. We consider Esther and Frank. The value of $Y$ for each of them is strictly between the value of $Y$ for George and the value of $Y$ for Dina. Therefore the three allocations of Esther, Frank, and Alice+Dina+George are identical. The rest of the proof is analogous to Option A.

Option C: Alice and George feel no envy. The value of $Y$ for all the singles is strictly between the value of $Y$ for Alice and the value of $Y$ for George. Therefore, the allocations of all five families must be identical. The rest of the proof is analogous to Theorem 13.

An interesting question that is left open by Theorems 12' and 14 is what happens when there are 3 or 4 families - does there always exist an allocation that is both $\mathrm{PE}$ and democratic-NE? 


\section{Related Work}

There are numerous papers about fair division in general and fair cake-cutting in particular. We mentioned some of them in the introduction. Here we survey some work that is more closely related to family-based fairness.

\subsection{Dividing indivisible items among groups}

In a contemporaneous and independent work, Manurangsi and Suksompong (2017) and Suksompong (2018) have studied the problem of fairly dividing discrete items among groups of agents. They focused on unanimous fairness. They proved that, in many cases, unanimously-fair allocations do not exist. These results complement our impossibility results for unanimous fairness in dividing a continuous resource. After the publication of their work and our working paper, we joined forces to study democratic-fair allocation of indivisible goods (Segal-Halevi and Suksompong 2018).

\subsection{Group-envy-freeness and on-the-fly coalitions}

Berliant et al (1992); Hüsseinov (2011); Todo et al (2011) study the concept of group-envy-freeness. They assume the standard model of fair division among individuals (and not among families). They define a group-envy-free division as a division in which no coalition of individuals can take the pieces allocated to another coalition with the same number of individuals and re-divide the pieces among its members such that all members are weakly better-off. Coalitions in cake-cutting are also studied by Dall'Aglio et al (2009); Dall'Aglio and Di Luca (2014).

In our setting, the families are pre-determined and the agents do not form coalitions on-the-fly. In an alternative model, in which agents are allowed to form coalitions based on their preferences, the family-fair-division problem becomes easier. For instance, it is easy to achieve a unanimous-FS division with connected pieces between two coalitions: ask each agent to mark its median line, find the median of all medians, then divide the agents to two coalitions according to whether their median line is to the left or to the right of the median-of-medians.

\subsection{Fair division with public goods}

In our setting, the piece given to each family is considered a "public good" in this specific family. The existence of fair allocations of homogeneous goods when some of the goods are public has been studied e.g. by Diamantaras (1992); Diamantaras and Wilkie (1994, 1996); Guth and Kliemt (2002). In these studies, each good is either private (consumed by a single agent) or public (consumed by all agents). In the present paper, each piece of land 
is consumed by all agents in a single family — a situation not captured by existing public-good models.

\subsection{Non-additive utilities}

As explained in Sections 4 and 5, the difficulty with unanimous-FS and democratic-FS is that the associated family-valuation functions are not additive. It is therefore interesting to compare our work to other works on cake-cutting with non-additive valuations.

Berliant et al (1992); Maccheroni and Marinacci (2003); Dall'Aglio and Maccheroni (2005) focus on sub-additive, or concave, valuations, in which the sum of the values of the parts is more than the value of the whole. These works are not applicable to our setting, because the family-valuations are not necessarily sub-additive - the sum of values of the parts might be less than the value of the whole (see the example in the beginning of Section 4).

Sagara and Vlach (2005); Dall'Aglio and Maccheroni (2009); Hüsseinov and Sagara (2013) consider general non-additive value functions. They provide pure existence proofs and do not say much about the nature of the resulting divisions (e.g, the number of connectivity components), which we believe is important in practical division applications.

$\mathrm{Su}$ (1999) presents a protocol for envy-free division with connected pieces which does not assume additivity of valuations. However, when the valuations are non-additive, there are no guarantees about the value per agent. In particular, with non-additive valuations, the resulting division is not necessarily FS.

Mirchandani (2013) suggests a division protocol for non-additive valuations using non-linear programming. However, the protocol is practical only when the resource to divide is a collection of a small number of homogeneous components, where the only thing that matters is what fraction of each component is allocated to each agent. In contrast, in our model the resource is a single heterogeneous good.

Finally, Berliant and Dunz (2004); Caragiannis et al (2011); Segal-Halevi et al (2015) study specific non-additive value functions which are motivated by geometric considerations (location, size and shape). The present paper contributes to this line of work by studying specific non-additive value functions which are motivated by a different consideration: handling the different preferences of family members. A possible future research topic is to find fair division rules that handle these considerations simultaneously, as both of them are important for fair division of land.

\section{References}

Alon N (1987) Splitting necklaces. Advances in Mathematics 63(3):247-253, DOI 10.1016/0001-8708(87)90055-7, URL http://dx.doi.org/10.1016/0001-8708(87)90055-7 Bade S, Segal-Halevi E (2018) Fair and Efficient Division Among Families, mimeo 
Barbanel JB, Brams SJ (2004) Cake division with minimal cuts: envy-free procedures for three persons, four persons, and beyond. Mathematical Social Sciences 48(3):251-269, DOI 10.1016/j.mathsocsci.2004.03.006, URL http://dx.doi.org/10.1016/j.mathsocsci.2004.03.006

Barbanel JB, Brams SJ (2014) Two-Person Cake Cutting: The Optimal Number of Cuts. The Mathematical Intelligencer 36(3):23-35, DOI 10.1007/s00283-013-9442-0, URL http://mpra.ub.uni-muenchen.de/34263/

Bentham J (1789) An Introduction to the Principles of Morals and Legislation (Dover Philosophical Classics). Dover Publications, URL http://www.worldcat.org/isbn/0486454525

Berliant M, Dunz K (2004) A foundation of location theory: existence of equilibrium, the welfare theorems, and core. Journal of Mathematical Economics 40(5):593-618, DOI 10.1016/s0304-4068(03)00077-6, URL http://dx.doi.org/10.1016/s0304-4068(03)000776

Berliant M, Thomson W, Dunz K (1992) On the fair division of a heterogeneous commodity. Journal of Mathematical Economics 21(3):201-216, DOI 10.1016/0304-4068(92)90001-n, URL http://dx.doi.org/10.1016/0304-4068(92)90001-n

Bogomolnaia A, Moulin H, Sandomirskiy F, Yanovskaya E (2017) Competitive division of a mixed manna. Econometrica 85(6):1847-1871, URL http://arxiv.org/abs/1702.00616, arXiv preprint $1702.00616,1702.00616$

Brams SJ, Taylor AD (1996) Fair Division: From Cake Cutting to Dispute Resolution. Cambridge University Press, Cambridge UK, URL http://www.worldcat.org/isbn/0521556449

Buchanan JM (1965) An economic theory of clubs. Economica pp 1-14, DOI 10.2307/2552442, URL http://dx.doi.org/10.2307/2552442

Caragiannis I, Lai JK, Procaccia AD (2011) Towards more expressive cake cutting. In: Proceedings of the Twenty-Second international joint conference on Artificial Intelligence (IJCAI'11), AAAI Press, IJCAI'11, pp 127-132, DOI 10.5591/978-1-57735-5168/ijcai11-033, URL http://www.cs.cmu.edu/ ./arielpro/papers/nonadd.ijcai11.pdf

Chambers CP (2005) Allocation rules for land division. Journal of Economic Theory 121(2):236-258, DOI 10.1016/j.jet.2004.04.008, URL http://dx.doi.org/10.1016/j.jet.2004.04.008

Chevaleyre Y, Dunne PE, Endriss U, Lang J, Lemaître M, Maudet N, Padget J, Phelps S, Rodriguez-Aguilar JA, Sousa P (2006) Issues in Multiagent Resource Allocation. Informatica 30(1):3-31, URL http://www.informatica.si/index.php/informatica/article/view/70

Cseh A, Fleiner T (2017) The complexity of cake cutting with unequal shares. URL http://arxiv.org/abs/1709.03152, 1709.03152

Dall'Aglio M, Di Luca C (2014) Finding maxmin allocations in cooperative and competitive fair division. Annals of Operations Research 223(1):121-136, DOI 10.1007/s10479-0141611-9, URL http://dx.doi.org/10.1007/s10479-014-1611-9

Dall'Aglio M, Maccheroni F (2005) Fair Division without Additivity. American Mathematical Monthly pp 363-365, DOI $10.2307 / 30037474$, URL http://dialnet.unirioja.es/servlet/articulo?codigo $=1140450$

Dall'Aglio M, Maccheroni F (2009) Disputed lands. Games and Economic Behavior 66(1):57-77, DOI 10.1016/j.geb.2008.04.006, URL http://eprints.luiss.it/727/1/Dall'Aglio_no.58_2007.pdf

Dall'Aglio M, Branzei R, Tijs S (2009) Cooperation in dividing the cake. TOP - the official journal of the Spanish Society of Statistics and Operations Research 17(2):417-432, DOI 10.1007/s11750-009-0075-6, URL http://dx.doi.org/10.1007/s11750-009-0075-6

Diamantaras D (1992) On equity with public goods. Social Choice and Welfare 9(2):141-157, DOI 10.1007/bf00187239, URL http://dx.doi.org/10.1007/bf00187239

Diamantaras D, Wilkie S (1994) A Generalization of Kaneko's Ratio Equilibrium for Economies with Private and Public Goods. Journal of Economic Theory 62(2):499-512, DOI 10.1006/jeth.1994.1028, URL http://dx.doi.org/10.1006/jeth.1994.1028

Diamantaras D, Wilkie S (1996) On the set of Pareto efficient allocations in economies with public goods. Economic Theory 7(2):371-379, DOI 10.1007/bf01213913, URL http://dx.doi.org/10.1007/bf01213913 
Dubins LE, Spanier EH (1961) How to Cut A Cake Fairly. The American Mathematical Monthly 68(1):1-17, DOI 10.2307/2311357, URL http://dx.doi.org/10.2307/2311357

Dvoretzky A, Wald A, Wolfowitz J (1951) Relations among certain ranges of vector measures. Pacific Journal of Mathematics 1(1):59-74, DOI 10.2140/pjm.1951.1.59, URL http://dx.doi.org/10.2140/pjm.1951.1.59

Even S, Paz A (1984) A Note on Cake Cutting. Discrete Applied Mathematics 7(3):285296, DOI 10.1016/0166-218x(84)90005-2, URL http://dx.doi.org/10.1016/0166$218 \mathrm{x}(84) 90005-2$

Foley DK (1967) Resource allocation and the public sector. YALE ECON ESSAYS 7(1):4598

Gamow G, Stern M (1958) Puzzle-math, first edition edn. Viking Adult, URL http://www.worldcat.org/isbn/0670583359

Guth W, Kliemt H (2002) Non-Discriminatory, Envy Free Provision of a Collective Good: A Note. Public Choice 111(1-2):179-184, URL http://search.proquest.com/openview/6a73b773b43c00706940913d6ecf5324/1?pqorigsite $=$ gscholar

Hüsseinov F (2011) A theory of a heterogeneous divisible commodity exchange economy. Journal of Mathematical Economics 47(1):54-59, DOI 10.1016/j.jmateco.2010.12.001, URL http://dx.doi.org/10.1016/j.jmateco.2010.12.001

Hüsseinov F, Sagara N (2013) Existence of efficient envy-free allocations of a heterogeneous divisible commodity with nonadditive utilities. Social Choice and Welfare pp 1-18, DOI 10.1007/s00355-012-0714-y, URL http://dx.doi.org/10.1007/s00355-012-0714-y

Maccheroni F, Marinacci M (2003) How to cut a pizza fairly: Fair division with decreasing marginal evaluations. Social Choice and Welfare 20(3):457-465, DOI 10.1007/s003550200192, URL http://dx.doi.org/10.1007/s003550200192

Manurangsi P, Suksompong W (2017) Asymptotic existence of fair divisions for groups. Mathematical Social Sciences 89:100-108

Mirchandani RS (2013) Superadditivity and Subadditivity in Fair Division. Journal of Mathematics Research 5(3):78-91, DOI 10.5539/jmr.v5n3p78, URL http://dx.doi.org/10.5539/jmr.v5n3p78

Moulin H (2004) Fair Division and Collective Welfare. The MIT Press, URL http://www.worldcat.org/isbn/0262633116

Procaccia AD (2015) Cake Cutting Algorithms. In: Brandt F, Conitzer V, Endriss U, Lang J, Procaccia AD (eds) Handbook of Computational Social Choice, Cambridge University Press, chap 13, pp 261-283, URL http://procaccia.info/papers/cakechapter.pdf

Robertson JM, Webb WA (1995) Approximating fair division with a limited number of cuts. Journal of Combinatorial Theory, Series A 72(2):340-344, DOI 10.1016/00973165(95)90073-x, URL http://dx.doi.org/10.1016/0097-3165(95)90073-x

Robertson JM, Webb WA (1998) Cake-Cutting Algorithms: Be Fair if You Can, 1st edn. A K Peters/CRC Press, URL http://www.worldcat.org/isbn/1568810768

Sagara N, Vlach M (2005) Equity and Efficiency in a Measure Space with Nonadditive Preferences: The Problem of Cake Division. Journal of Economic Literature 90:1, URL http://web.econ.keio.ac.jp/staff/maruyama/rcme/seminar/abst050523.pdf

Segal-Halevi E (2018) Cake-Cutting with Different Entitlements: How Many Cuts are Needed? ArXiv preprint 1803.05470

Segal-Halevi E, Nitzan S (2016) Envy-Free Cake-Cutting among Families. URL http://arxiv.org/abs/1607.01517, arXiv preprint 1607.01517

Segal-Halevi E, Suksompong W (2018) Democratic Fair Allocation of Indivisible Goods. In: Proceedings of the 27th International Joint Conference on Artificial Intelligence (IJCAI '18), URL http://arxiv.org/abs/1709.02564, arXiv preprint 1709.0256

Segal-Halevi E, Sziklai BR (2018) Monotonicity and Competitive Equilibrium in Cakecutting. Economic Theory URL https://doi.org/10.1007/s00199-018-1128-6, arXiv preprint 1510.05229

Segal-Halevi E, Hassidim A, Aumann Y (2015) Envy-Free Cake-Cutting in Two Dimensions. In: Proceedings of the 29th AAAI Conference on Artificial Intelligence (AAAI-15), pp 1021-1028, URL http://www.aaai.org/ocs/index.php/AAAI/AAAI15/paper/view/9656, arXiv preprint 1609.03938 
Shishido H, Zeng DZ (1999) Mark-Choose-Cut Algorithms For Fair And Strongly Fair Division. Group Decision and Negotiation 8(2):125-137, DOI 10.1023/a:1008620404353, URL http://dx.doi.org/10.1023/a:1008620404353

Steinhaus H (1948) The problem of fair division. Econometrica 16(1):101-104, URL http://www.jstor.org/stable/1914289

Stromquist W (1980) How to Cut a Cake Fairly. The American Mathematical Monthly 87(8):640-644, DOI 10.2307/2320951, URL http://dx.doi.org/10.2307/2320951

Stromquist W, Woodall DR (1985) Sets on which several measures agree. Journal of Mathematical Analysis and Applications 108(1):241-248, DOI 10.1016/0022-247x(85)90021-6, URL http://dx.doi.org/10.1016/0022-247x(85)90021-6

Su FE (1999) Rental Harmony: Sperner's Lemma in Fair Division. The American Mathematical Monthly 106(10):930-942, DOI 10.2307/2589747, URL http://www.math.hmc.edu/ su/papers.dir/rent.pdf

Suksompong W (2018) Approximate maximin shares for groups of agents. Mathematical Social Sciences Http://doi.org/10.1016/j.mathsocsci.2017. 09.004

Todo T, Li R, Hu X, Mouri T, Iwasaki A, Yokoo M (2011) Generalizing envy-freeness toward group of agents. In: IJCAI ProceedingsInternational Joint Conference on Artificial Intelligence, vol 22, p 386, URL https://www.aaai.org/ocs/index.php/IJCAI/IJCAI11/paper/viewFile/3255/3382

Webb WA (1997) How to cut a cake fairly using a minimal number of cuts. Discrete Applied Mathematics 74(2):183-190, DOI 10.1016/s0166-218x(96)00032-7, URL http://dx.doi.org/10.1016/s0166-218x(96)00032-7 